\title{
Safety and tolerability of the antioxidant OPC-14117 in HIV-associated cognitive impairment
}

\author{
The Dana Consortium on the Therapy of HIV Dementia and Related Cognitive Disorders*
}

\begin{abstract}
Article abstract-Cognitive impairment is a common and disabling complication of advanced HIV infection. Antiretroviral agents are the only proven therapies currently used for the treatment of HIV dementia, but the response to these agents is frequently unsatisfactory, short-lived, or complicated by intolerable side effects. We hypothesized that OPC14117, a lipophilic antioxidant that acts to scavenge superoxide anion radicals, might ameliorate the toxic interactions between HIV infected macrophages and neurons. We conducted a double-blind, placebo-controlled, randomized clinical trial to assess the safety and tolerability of OPC-14117 $240 \mathrm{mg}$ per day. All 30 patients enrolled (15 per group) had cognitive impairment based on performance on neuropsychological tests. The primary outcome was tolerability of the study drug as measured by the proportion of subjects able to complete the study on their assigned dosage of experimental medication. Overall OPC-14117 was as well tolerated as placebo. Five subjects withdrew because of adverse experiences (two placebo, three OPC-14117). The OPC-14117-treated group had better scores on a clinical global impression scale, compared with the placebo group. There were trends toward improvement in the cognitive test scores; however, these changes were not statistically significant. These results demonstrate that this antioxidant intervention is well tolerated in cognitively impaired patients with advanced HIV infection, and suggest that a larger efficacy trial to assess the impact of OPC-14117 on cognitive performance is warranted.
\end{abstract}

NEUROLOGY 1997;49:142-146

Cognitive impairment is a common and disabling complication of advanced HIV infection. The term "HIV-associated cognitive motor complex" (HACMC) encompasses the constellation of signs and symptoms that includes not only cognitive impairment but also motor dysfunction and behavioral change. ${ }^{1}$ The most severe form of this entity, the HIV-1-associated dementia complex (HADC), is a progressive disorder with a mean survival of 6 months, and with mortality usually attributable to the effects of the neurologic disease. ${ }^{2}$ The annual incidence of HADC among patients with AIDS is 7\%, and investigators estimate that 20 to $30 \%$ of all individuals with AIDS will develop cognitive impairment. ${ }^{2}$

Antiretroviral agents are currently used for treatment of HACMC, but the treatment response is frequently unsatisfactory, short-lived, or associated with intolerable side effects. Some antiretroviral agents (e.g., didanosine) have relatively poor CNS penetrance $^{3}$ and hence may be less effective for treating cognitive impairment. Open-label studies with zidovudine in demented individuals showed promising improvements in clinical functioning and neuropsychologic performance. ${ }^{4}$ Larger blinded studies suggested that zidovudine improved neuropsychological function in the short term among individuals with AIDS, but without overt HADC. ${ }^{5}$ The only placebo-controlled clinical trial of drug treatment for HADC showed that zidovudine in doses of 1,000 to $2,000 \mathrm{mg}$ per day improved cognitive function. The beneficial effects were evident within 1 to 2 months of the initiation of zidovudine. ${ }^{6}$

Our understanding of the mechanisms of neuronal injury has evolved, ${ }^{7,8}$ and clinical trials have evaluated novel interventions in HIV-related cognitive impairment. Effective therapy for HADC may depend not only on targeting the virus but on blocking "downstream" mechanisms of cell injury. Such interventions may target the excitatory amino acid receptor-mediated or the voltage-sensitive influx of calcium ions, cytokines (including tumor necrosis factor, interleukin 6), lipid inflammatory mediators (including arachidonic acid and platelet activating factor), and free radical species. ${ }^{8}$

We hypothesized that OPC-14117, a lipophilic compound with structural homology to vitamin $\mathrm{E}$, which in turn acts to scavenge superoxide anion radicals, may interfere with the toxic interactions be-

\footnotetext{
* All participants are listed in the Appendix.

Sponsored primarily by the Charles A. Dana Foundation and by the NIH General Clinical Research Centers at Rochester (RR00044), Johns Hopkins University (RR00722), and Columbia University (RR00645), and by the additional support of NS26643 at Johns Hopkins University and P50-MH43520 at the HIV Center for Clinical and Behavioral Studies, NYS Psychiatric Institute, OPC-14117 and matching placebo were provided by Otsuka America Pharmaceuticals, Inc., Rockville, $\mathrm{MD}$

Presented in abstract form at the Neuroscience of HIV conference, Paris, France, March 1996.

Received September 5, 1996. Accepted in final form January 16, 1997.

Address correspondence and reprint requests to Dr. Karl Kieburtz, University of Rochester, Department of Neurology, 601 Elmwood Ave., Box 673, Rochester, NY 14642.
} 
tween HIV-infected macrophages and neurons. We were particularly interested in the role of oxygen radicals since they have been implicated in the pathogenesis of neurodegenerative disease, and Coyle and Puttfarken ${ }^{9}$ suggested that glutamate neurotoxicity is mediated in part by free radicals. Although OPC-14117 has generally been well tolerated by patients with neurologic disorders, it may cause elevations in serum liver transaminase levels. ${ }^{10}$ We conducted a randomized, double-blind, placebo-controlled clinical trial primarily to assess the safety and tolerability of OPC-14117 in patients with HIV-related cognitive impairment, and secondarily, to assess the impact of OPC-14117 on cognitive performance.

Methods. Organization. This multicenter study was organized by the Charles A. Dana Foundation Consortium on the Therapy of HIV Dementia and Related Cognitive Disorders (University of Rochester, Columbia University, Johns Hopkins University) and sponsored by the Charles A. Dana Foundation (New York, NY). The study was approved by the Institutional Review Board at each center. An independent Safety Monitoring Committee periodically reviewed the safety of the study.

Recruitment and enrollment. Thirty eligible subjects were enrolled in the trial. Patients who had HIV infection, evidence of cognitive impairment, and who were taking a stable antiretroviral regimen for 6 weeks prior to randomization were eligible. Cognitive impairment was defined as performing at or below one standard deviation from the mean on at least two neuropsychological tests, or two standard deviations below the mean on at least one test.11 Neuropsychological test scores for subjects with 12 years of education or less were compared with norms established by the ALIVE study, ${ }^{12}$ and for subjects with more than 12 years of education norms established by the Multicenter AIDS Cohort Study ${ }^{13}$ were used. Patients were excluded if they had a history of opportunistic CNS infection, severe premorbid psychiatric illness likely to interfere with protocol compliance, history of chronic neurologic disorder unrelated to HIV infection, serum liver transaminase values greater than or equal to three times the upper limit of normal or a history of alcoholism within the past 6 months, or any other clinically significant condition or laboratory abnormality that, in the investigator's opinion, would interfere with the subject's ability to participate in the study. The active use of illicit drugs was not exclusionary; however, individuals were screened for the stability of drug use and the likelihood that such use would continue throughout the study. Subjects were instructed that during the study they could average only one alcoholic drink per day and should not exceed two alcoholic drinks per day.

After informed consent was obtained, subjects were randomly assigned at the baseline evaluation to receive either OPC-14117 or placebo. The computer-generated randomization plan included stratification by center and blocking. The OPC-14117-treated subjects received $120 \mathrm{mg}$ daily for the initial 6 weeks of the study and $240 \mathrm{mg}$ daily for the remaining 6 weeks. Assignment to treatment was performed through a call-in computer enrollment module that maintained the blindness of treatment assignment for all subjects and staff involved in the study.

Therapy and follow-up. The subjects took $60-\mathrm{mg}$ tablets of OPC-14117 or matching tablets of placebo (Otsuka America Pharmaceutical Inc., Rockville, MD) orally, twice daily, approximately 12 hours apart. Experimental treatments started on the morning after randomization. For the initial 6 weeks of treatment the dosage was $60 \mathrm{mg}$ twice daily. The maintenance dosage of $120 \mathrm{mg}$ twice daily was started at the sixth week of the study. All subjects took two tablets twice daily throughout the study. The appropriate combination of $60 \mathrm{mg}$ OPC- 14117 and matching placebo tablets was used to achieve the appropriate dosage. Subjects were re-evaluated at $2,4,8$, and 12 weeks after randomization. At each visit, subjects were assessed for adverse clinical experiences. A battery of safety surveillance laboratory tests was performed at each visit and included urinalysis, hematology, and serum chemistry profiles. In addition, serum liver transaminase levels (aspartate aminotransferase, alanine aminotransferase, and alkaline phosphatase) were collected at $1,3,5,6,7$, and 10 weeks after randomization. Clinical assessments performed at each visit included vital signs, Karnofsky Performance scale, ${ }^{14}$ and pill counting to assess compliance. Neuropsychological evaluation included the Rey Auditory Verbal Learning Test, ${ }^{15}$ Digit Symbol Test, ${ }^{16}$ Grooved Pegboard (dominant and nondominant hands), ${ }^{17}$ and timed gait, and was performed at baseline and at 4 and 12 weeks. A functional assessment, including activities of daily living (ADLs) on the Personal Self Maintenance Scale ${ }^{18}$ (range: 7-14 [least impaired]), instrumental ADLs of Lawton and Brody $^{19}$ (range 9-27 [least impaired]), scales of the Medical Outcomes Study, ${ }^{20}$ and an assessment of mood using the Center for Epidemiologic Studies Depression Scale $(\text { CES-D })^{21}$ were performed at baseline and at 4,8 , and 12 weeks. A neurologic examination, ${ }^{6}$ global impression score, CD4+ lymphocyte counts, and a serum beta-2 microglobulin level were performed at baseline and at 12 weeks. The global impression score rated the cognitive ability of subjects as normal (0), mild impairment (1), moderate impairment (2), or severe impairment (3).

The investigator was permitted to halve the assigned dosage of experimental medication if the subject developed persistent or recurrent adverse experiences judged to be of moderate (sign or symptom intense enough to interfere with usual activity) or severe (sign or symptom interferes significantly with ability to do work or usual activity) intensity. If the adverse experience improved, the full number of tablets was resumed. In addition, if the subject developed elevated serum liver enzyme levels, an algorithm of dosage reduction or suspension was employed. Subjects were withdrawn from the study if an adverse experience was judged to be severe and persistent.

Outcome measures. The primary outcome measure of the study was whether or not the subject completed the study at the originally assigned dosage of experimental medications, regardless of whether dosage halving or suspension occurred during the study. A secondary measure of tolerability was whether or not the subject completed the study. Measures of safety included frequencies of adverse experiences and abnormal results on laboratory tests and changes over time in laboratory tests and vital signs. Measures of efficacy included 4- and 12-week changes from 
baseline in neuropsychological test results, global impression scores, and measures of function and mood.

Sample size considerations. The sample size of 15 subjects per group was chosen to provide approximately $90 \%$ power to detect a $45 \%$ difference in tolerability ( $95 \%$ versus $50 \%$ ) between the placebo and OPC-14117 groups using a one-sided Fisher's exact test at the $5 \%$ level of significance. If approximately $50 \%$ of the subjects assigned to the OPC-14117 group could tolerate the assigned dosage, then this medication was considered a reasonable candidate for further investigation, due to the inexorable progression of HIV dementia and the lack of any currently available effective treatment other than zidovudine.

Statistical methods. In accordance with the intentionto-treat principle, all 30 subjects were included in all statistical analyses. The proportion of subjects unable to tolerate the experimental medications was compared among the groups using a one-sided Fisher's exact test, modified using the mid $p$-value. ${ }^{22}$ Similar analyses were performed for the secondary tolerability measures and for the incidences of adverse experiences and abnormal laboratory tests.

Analyses of the efficacy variables used a two-way analysis of variance model, with the 12-week change from baseline for the variable of interest as the dependent variable, treatment group as the factor of interest, and center as the stratification factor. F-tests were performed for significance of the treatment effect, and $95 \%$ confidence intervals for the treatment effect were also computed. Similar analyses were performed for changes in laboratory test results and vital signs. Changes in global impression scores were compared between the groups using Fisher's exact test. For subjects who prematurely dropped out of the study, the last available observation recorded for the subject was carried forward for all subsequent visits for purposes of the primary statistical analyses. Separate additional analyses were performed that included only subjects who did not prematurely drop out of the study; however, the results of these analyses did not differ substantially from those of the primary analyses and hence are not reported here.

Results, Comparability of treatment groups. The treatment groups were very similar at baseline with regard to demographic and clinical variables (table 1 ). The average duration of HIV infection was approximately 5 years and the average CD4+ lymphocyte count was approximately $230 / \mathrm{mm}^{3}$. The global cognitive deficit ratings of mild and moderate correspond with the HADC stages 0.5 and $1 .^{6}$

Tolerability of experimental medications. There were no significant differences between the placebo group and the OPC-14117 group regarding the primary or secondary measures of tolerability. In the placebo group nine of 15 subjects $(60 \%)$, compared with seven of 15 subjects $(47.7 \%)$ in the OPC-14117 group, completed the study on the originally assigned dosage. In the placebo group 10 of 15 subjects $(66.7 \%)$, compared with eight of 15 subjects in the OPC-14117 group (53.3\%) completed the study on any dosage. Dosage halving occurred in two subjects due to increases in serum liver enzyme levels, one in the placebo group and one in the OPC-14117 group. For the subjects dropping out within the placebo group, two discontinued participation because of adverse experiences (diarrhea, parotid tumor) and three withdrew consent. Within the OPC14117 group, three subjects experienced adverse experi-
Table 1 Baseline characteristics

\begin{tabular}{|c|c|c|}
\hline & $\begin{array}{l}\text { Placebo } \\
(\mathrm{n}=15)\end{array}$ & $\begin{array}{l}\text { OPC-14117 } \\
(\mathrm{n}=15)\end{array}$ \\
\hline Gender (male/female) & $12 / 3$ & $13 / 2$ \\
\hline Race (white/black/other) & $8 / 6 / 1$ & $9 / 5 / 1$ \\
\hline Age (years) & $42.9 \pm 10.7$ & $40.5 \pm 9.4$ \\
\hline Education (years) & $14.1 \pm 3.2$ & $12.9 \pm 2.8$ \\
\hline $\begin{array}{l}\text { Estimated duration HIV (+) } \\
\text { (years) }\end{array}$ & $4.9 \pm 3.2$ & $5.6 \pm 3.0$ \\
\hline Weight (kg) & $81.4 \pm 21.1$ & $81.3 \pm 23.1$ \\
\hline CD4 + lymphocyte count $/ \mathrm{mm}^{3}$ & $229 \pm 195$ & $238 \pm 173$ \\
\hline Karnofsky score & $81.8 \pm 14.9$ & $88.9 \pm 9.0$ \\
\hline $\begin{array}{l}\text { Global cognitive deficit (mild/ } \\
\text { moderate) }\end{array}$ & $6 / 9$ & $10 / 5$ \\
\hline Instrumental ADL score & $21.8 \pm 3.4$ & $23.0 \pm 1.2$ \\
\hline Self maintenance ADL score & $17.4 \pm 1.5$ & $17.9 \pm 0.3$ \\
\hline Role function score & $11.1 \pm 2.1$ & $11.2 \pm 2.1$ \\
\hline Physical function score & $20.3 \pm 5.9$ & $22.4 \pm 3.9$ \\
\hline \multicolumn{3}{|l|}{ Rey AVM (number correct) } \\
\hline Total & $45.1 \pm 9.8$ & $43.1 \pm 7.6$ \\
\hline Recall after interference & $8.5 \pm 2.7$ & $7.8 \pm 4.1$ \\
\hline Delayed recall & $8.1 \pm 3.1$ & $7.5 \pm 3.7$ \\
\hline Symbol digit (number correct) & $43.1 \pm 15.4$ & $44.9 \pm 10.7$ \\
\hline \multicolumn{3}{|l|}{ Grooved pegboard (sec) } \\
\hline Dominant & $92.6 \pm 24.1$ & $79.4 \pm \mathbf{1 7 . 5}$ \\
\hline Nondominant & $110.0 \pm 32.0$ & $85.3 \pm 17.2$ \\
\hline Timed gait (sec) & $13.6 \pm 5.6$ & $10.8 \pm 3.0$ \\
\hline CES-D & $18.1 \pm 8.7$ & $16.5 \pm 5.3$ \\
\hline
\end{tabular}

Values are mean $\pm S D$ unless otherwise indicated.

AVM = auditory verbal memory; CES-D = Center of Epidemiologic Studies-Depression Scale.

ences that prompted withdrawal (rash, diarrhea, renal impairment) and four subjects withdrew consent. Those subjects who withdrew consent either elected to discontinue participation or failed to return for follow-up. No adverse experiences were known to be the cause for withdrawing consent. A total of 60 different adverse experiences was reported among the study subjects. There was no difference in the frequency of adverse experiences among the two treatment groups. The most common adverse experiences were fatigue (five placebo, three OPC14117), diarrhea (two placebo, three OPC-14117), nausea (three placebo, one OPC-14117) and cough (three placebo, 0 OPC-14117). All of the adverse experiences reported to be of severe intensity (metabolic encephalopathy, parotid tumor, diarrhea, fatigue, pneumonia, nausea) occurred in the placebo-treated group.

There were no significant differences between the treatment groups regarding changes in laboratory tests. The mean compliance rate was $84.1 \%$ in the placebo group and $64.1 \%$ in the OPC-14117 group. When excluding subjects who dropped out prematurely, the compliance in the OPC14117 group was $78.4 \%$ and compliance in the placebo group was $83.1 \%$. No illicit drug use was reported during the trial, but information was given voluntarily. 
Table 2 Mean changes from baseline to 12 weeks in neuropsychological test change scores

\begin{tabular}{|c|c|c|c|c|c|}
\hline & Placebo $(n=15)$ & OPC-14117 $(n=15)$ & Treatment effect* & $95 \% \mathrm{CI}$ & $p$ Value \\
\hline \multicolumn{6}{|l|}{ Rey Auditory Verbal Memory } \\
\hline Total number correct & $-2.0(9.9)$ & $0.3(9.6)$ & 2.1 & $(-5.2,9.4)$ & 0.56 \\
\hline Recall after interference & $-0.9(2.0)$ & $0.8(3.5)$ & 1.6 & $(-0.6,3.9)$ & 0.14 \\
\hline Delayed recall & $-0.9(2.0)$ & $0.6(3.4)$ & 1.4 & $(-0.7,3.6)$ & 0.18 \\
\hline Symbol digit (number correct) & $-0.3(6.4)$ & $0.1(4.1)$ & 0.2 & $(-3.9,4.3)$ & 0.92 \\
\hline \multicolumn{6}{|l|}{ Grooved pegboard (sec) } \\
\hline Dominant & $0.4(30.6)$ & $-3.3(13.7)$ & -3.7 & $(-22.1,14.6)$ & 0.68 \\
\hline Nondominant & $6.4(30.1)$ & $-5.0(20.8)$ & -11.7 & $(-30.7,7.3)$ & 0.22 \\
\hline Timed gait (sec) & $-0.8(2.6)$ & $0.3(2.1)$ & 1.2 & $(-0.7,3.1)$ & 0.20 \\
\hline
\end{tabular}

Values are mean (standard deviation); positive values indicate improvement.

* Treatment effect is the difference in mean change between the OPC-14117 group and the placebo group, adjusted for investigator effects in a two-way analysis of variance model. See text for details.

Clinical measures. The 12-week changes from baseline in the efficacy measures, with the last available observation carried forward for subjects not completing the study, are listed in table 2. Although there were no significant differences between the groups regarding these mean changes, there were trends toward improvement in the Rey Auditory Verbal Learning Recall, Delayed Recall Subscales, and in the timed gait test, favoring the OPC group. The 4-week changes were similar (data not shown). There was a difference in the changes in global impression of cognitive ability scores at 12 weeks with three subjects worsened, 11 stable, and one improved in the OPC- 14117 group, compared with nine subjects worsened, five stable, and one improved in the placebo group (nominal $p=0.03$ ). There were no significant differences between the groups on mean changes in function, mood, CD4 lymphocyte count, and beta-2-microglobulin levels.

Discussion. Our study demonstrates that OPC14117 is generally well tolerated in subjects with cognitive impairment and advanced HIV infection. There were no significant differences between the treatment groups with respect to the predetermined tolerability measures. The dropout rate in the placebo group was somewhat higher than we anticipated, which adversely affected the power of the study to detect group differences in tolerability. Nevertheless, the observed rates of intolerability were very similar in the two groups, as was the profile of adverse experiences. The dropout rate in the overall study sample probably reflects the severity of the underlying systemic disease in these patients. The somewhat lower compliance rate in the OPC group may have enhanced its apparent tolerability.

Our results are particularly encouraging since prior studies of OPC-14117 found elevations in liver transaminase levels. ${ }^{10}$ In this study, there were no differences between the two treatment groups in the frequency of liver function test abnormalities nor in the mean changes of liver function tests over time, and no severe adverse experiences in the OPC-14117 treated group.
Although the study was not primarily designed to detect clinical improvement, the global impression scores were better in the OPC-14117 treated group compared with placebo. Furthermore, analyses of the neuropsychologic test scores raise the possibility that OPC-14117 may exert a mild beneficial effect on tests of memory and timed motor function. However, the magnitude of the change observed in the neuropsychological test scores was small and not statistically significant and was not associated with improvements in mood or functional capacity. There were no significant changes in the depression rating scale or in the measures of daily function, although the functional measures may not be as sensitive to short-term changes as other measures. Longer-term improvements in cognitive performance would likely lead to functional benefit, as cognitive and functional capacity are correlated in HIV dementia. ${ }^{11}$

Our study differs from previous trials that have assessed the impact of interventions in patients with $\mathrm{HADC}$ in that we enrolled patients with less-severe cognitive impairment (HADC stages 0.5 and 1.0). ${ }^{6}$ Patients in the early stages of HIV-associated cognitive impairment may be more likely to tolerate participation in longer-term clinical trials and may benefit the most from symptomatic improvement as these patients might potentially have "reversible" neurologic disease, whereas patients with severe dementia might have irreversible neuronal loss. Our study is also different in that there was a high proportion of women (16\%) and ethnic minorities (43\%). In addition we allowed participation of subjects with a history of, or current, illicit drug use. No subjects reported illicit drug use during the trial, but unreported drug use may have influenced cognitive test performance. The ethnic and gender heterogeneity of the study participants enhances the generalizability of our findings.

We demonstrated that OPC-14117 is safe and well tolerated by HIV-infected patients with moderate to severe immunosuppression. Further investigations 
are warranted to assess the influence of OPC-14117 on the clinical features of HIV-related cognitive impairment. In addition, future studies may rationally assess the effect of the combination of antioxidant interventions such as OPC-14117 with antiviral agents or other agents that interfere with the cascade of neurotoxic events.

\section{Acknowledgments}

The authors thank the scientific advisory committee (Thomas Chase, MD, Mary Foulkes, $\mathrm{PhD}$, Stephen Foster, Mindy Fullilove, MD, Robert Hultz, Carl Leventhal, MD, and Donna Mildvan, MD) for the critical review and scientific guidance of this research study.

Appendix. Dana Consortium on the Therapy of HIV Dementia and Related Cognitive Disorders:

University of Rochester. Karl Kieburtz, MD, MPH; Giovanni Schifitto, MD; Michael McDermott, PhD; Moonseong Heo, PhD; Carrie Irvine, BS; Donna Palumbo, PhD; Connie Orme; Carol Zimmerman, RN; Harris Gelbard, $\mathrm{MD}, \mathrm{PhD}$; Charlyne Hickey, RN; Cindy Casaceli; Barry Guthrie; Lisa Rumfola; Leon Epstein, MD.

Columbia University. Karen Marder, MD, MPH; Gayatri Ramachandran, MD; Steven Albert, PhD; George Dooneief, MD, MPH; Yaakov Stern, PhD; George Todak, MSW; Ronda Friedman-Clouse, RN; Carmen Polanco; Thomas Winston; Richard Mayeux, MD, MSE.

Johns Hopkins. Gerald Dal Pan, MD, MPH; Ola Selnes, PhD; Ned Sacktor, MD; Deborah Hasenauer, MA; Deneen Esposito; Letitia Nance-Sproson, MHA; Justin C. McArthur, MS, BS, MPH.

Safety Monitoring Committee. Ira Shoulson, MD (Chair); David Oakes, PhD; Richard Reichman, MD.

Dana Foundation. James O'Sullivan.

\section{References}

1. Janssen RS, Cornblath DR, Epstein LG, et al. Nomenclature and research case definitions for neurological manifestations of human immunodeficiency virus type-1 (HIV-1) infection. Report of a Working Group of the American Academy of Neurology AIDS Task Force. Neurology 1991;41:778-785.

2. McArthur JC, Hoover DR, Bacellar H, et al. Dementia in AIDS patients: incidence and risk factors. Neurology 1993;43: 2245-2252.

3. Yarchoan R, Pluda JM, Thomas RV, et al. Long-term toxicity/ activity profile of $2^{\prime}, 3^{\prime}$-dideoxyinosine in AIDS and AIDSrelated complex. Lancet 1990;336:526-529.
4. Yarchoan R, Berg G, Brouwers P, et al. Response of humanimmunodeficiency-virus-associated neurological disease to $3^{\prime}$ azido-3'-deoxythymidine. Lancet 1987;1:132-135.

5. Schmitt FA, Bigley JWS, McKinnis R, et al. Neuropsychological outcome of zidovudine (AZT) treatment of patients with AIDS and AIDS-related complex. N Engl J Med 1988;319: $1573-1578$.

6. Sidtis JJ, Gatsonis C, Price RW, et al. Zidovudine treatment of the AIDS dementia complex: results of a placebo-controlled trial. Ann Neurol 1993;33:343-349.

7. Epstein LG, Gendelman HE. Human immunodeficiency virus type 1 infection of the nervous system: pathogenetic mechanisms. Ann Neurol 1993;33:429-436.

8. Lipton SA, Gendelman HE. The dementia associated with the acquired immunodeficiency syndrome. N Engl J Med 1995; 332:934-940.

9. Coyle JT, Puttfarken P. Oxidative stress, glutamate and neurodegenerative disorders. Science 1993;262:698-700.

10. Huntington Study Group (F Marshall primary author). Safety and tolerability of the free-radical scavenger OPC-14117 in Huntington's disease [abstract]. Mov Disord 1996; 11(suppl 1): 59.

11. Dana Consortium on Therapy For HIV Dementia and Related Cognitive Disorders. Clinical Confirmation of the American Academy of Neurology Algorithm for HIV Associated Cognitive Motor Disorder. Neurology 1996;47:1247-1253.

12. Concha M, Selnes OA, McArthur J, et al. Normative data for a brief neuropsychologic test battery in a cohort of injecting drug users. Int J Addict 1995;30:823-841.

13. Selnes OA, Jacobson L, Machado AM, et al. Normative data for a brief neuropsychological screening battery. Percept Mot Skills 1991;73:539-550.

14. Karnofsky DA, Abelman WH, Carver LF, et al. The use of nitrogen mustards in the palliative treatment of carcinoma. Cancer 1948;1:634-656.

15. Rey A. L'examen psychologique dans les cas d'encephalopathie traumatique. Arch Psychologie 1941;28:286-340.

16. Wechsler D. Wechsler Adult Intelligence Scale Revised. New York, NY: The Psychological Corporation, 1981.

17. Klove H. Clinical neuropsychology. Med Clin North Am 1963; 46:1647-1658.

18. Katz S, Ford A, Moskowitz R, et al. Studies of illness in the aged: the index of ADL. JAMA 1963;183:914-919.

19. Lawton MP, Brody EM. Assessment of older people: selfmaintaining and instrumental activities of daily living. Gerontologist 1969;9:179-186.

20. Stewart $\mathrm{AL}$, Ware JE. Measuring function and well-being: The Medical Outcomes Study Approach. Durham, NC: Duke University Press, 1993.

21. Radloff LL. The CES-D: a self-report depression scale for research in the general population. Appl Psychol Meas 1977;1: 385-401.

22. Agresti A. Categorical data analysis. New York: Wiley, 1990. 